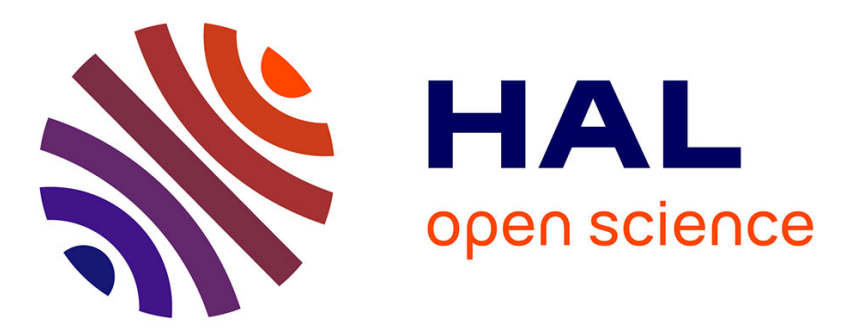

\title{
Analysis of Carbon and Nitrogen in High-Purity $\alpha$-Iron by Magnetic After-Effect Measurements
}

\author{
M. Hirscher, F. Walz, M. Weller
}

\section{To cite this version:}

M. Hirscher, F. Walz, M. Weller. Analysis of Carbon and Nitrogen in High-Purity $\alpha$-Iron by Magnetic After-Effect Measurements. Journal de Physique IV Proceedings, 1995, 05 (C7), pp.C7-175-C7-180. 10.1051/jp4:1995718 . jpa-00254011

\section{HAL Id: jpa-00254011 https://hal.science/jpa-00254011}

Submitted on 1 Jan 1995

HAL is a multi-disciplinary open access archive for the deposit and dissemination of scientific research documents, whether they are published or not. The documents may come from teaching and research institutions in France or abroad, or from public or private research centers.
L'archive ouverte pluridisciplinaire HAL, est destinée au dépôt et à la diffusion de documents scientifiques de niveau recherche, publiés ou non, émanant des établissements d'enseignement et de recherche français ou étrangers, des laboratoires publics ou privés. 


\title{
Analysis of Carbon and Nitrogen in High-Purity $\alpha$-Iron by Magnetic After-Effect Measurements
}

\author{
M. Hirscher*, F. Walz* and M. Weller** \\ Max-Planck-Institut für Metallforschung, \\ * Institut fiir Physik, Heisenbergstr. 1, 70569 Stuttgart, Germany \\ ** Institut für Werkstoffwissenschaft, Seestr. 92, 70174 Stuttgart, Germany
}

\begin{abstract}
Magnetic after-effect measurements (MAE) on carbon or nitrogen doped $\alpha$-iron show characteristic relaxation maxima. The microscopic nature of the MAE is briefly discussed and the measuring technique applying an LC oscillator is explained. Measurements on high-purity iron after different refinements are used to show the analysing sensitivity and limits. The results are compared to chemical analysis and internal friction measurements.
\end{abstract}

\section{INTRODUCTION}

The analysis of interstitially dissolved impurity atoms in high-purity metals below concentration levels of 100 at.ppm is very difficult by applying standard chemical methods [1]. Additional drawbacks of chemical methods are that they are usually destructive to the specimen and consume rather long analysing time. Recent developments of these methods enable the determination of trace elements in high-purity metals $[1,2]$, but the bottleneck is still the sample pretreatment, which requires extreme care to avoid problems, e.g. surface contamination. More sophisticated techniques including nuclear methods are very promising to achieve high accuracy, but need high-cost equipment [3].

Mechanical and magnetic relaxation techniques are very sensitive to diffusional jumps of interstitially dissolved atoms between neighbouring interstitial sites and non-destructive, i.e. avoid complications of contaminations from surface or solvents. The anelastic relaxation technique or internal friction is extensively discussed in [4-6]. For ferromagnetic materials the technique of magnetic after-effect (MAE) [7-9] can be applied. This technique has been successfully used to study e.g. radiation defects [10] or hydrogen [11] in metals. In these investigations the major aim was to determine the activation parameters of diffusion, but not quantitatively the concentration. In this paper the analysis of carbon and nitrogen in high-purity $\alpha$-iron by MAE will be discussed.

\section{THEORETICAL BACKGROUND}

Already in 1937 Richter reported on magnetic after-effects he observed in carbon steel [12]. In systematic measurements he described the time and temperature dependence and clearly distinguished this MAE from the irreversible so-called Jordan after-effect. The atomistic nature of this Richter MAE was first explained by Snoek [13] who assigned the effect to the reorientation of the anisotropy axis of interstitially dissolved carbon atoms. In $\alpha$-iron the carbon atoms are located on octahedral interstitial sites centered on the edges of the iron unit cell. This defect possesses a tetragonal symmetry with a four-fold $\langle 100\rangle$ axis parallel to the cube edge. In order to reorient the symmetry axis the carbon atom has to jump to a neighbouring interstitial site. The origin of this orientation MAE is the interaction energy $E_{i}$ of the defect on the $i$-th site

$$
E_{i}=\epsilon \gamma_{i}^{2},
$$

where $\gamma_{i}$ is the cosine between the $i$-th $<100>$ axis and the direction of the spontaneous magnetization and $\epsilon$ an effective interaction constant including the magnetocrystalline and the magnetoelastic interaction. By 
studying the time dependence of magnetic anisotropy and of length change, De Vries et al. [14] determined experimentally a positive interaction constant, i.e. edges with $<100>$ directions possessing the largest angle to the magnetization are preferentially occupied. These results are in agreement with calculations of Néel [15] who showed that the magnetocrystalline interaction is more effective than the magnetostrictive.

The reorientation of the anisotropy axis is due to a thermally activated jump to a neighbouring interstitial site, where the temperature dependence of the relaxation time $\tau$ can be described by an Arrhenius law

$$
\tau(T)=\tau_{o} \exp [Q / k T],
$$

with a pre-exponential factor $\tau_{o}$, an activation enthalpy $Q$, and the Boltzmann constant $k$. In this case of a Debye process, the time dependence of the reluctivity $r(t, T)=1 / \chi_{a}(t, T)$, where $\chi_{a}(t, T)$ denotes the initial magnetic susceptibility, is given by

$$
r(t, T)=r(0)+[r(\infty, T)-r(0, T)] G(t, \tau) \text { with } \quad G(t, \tau)=1-\exp [-t / \tau],
$$

where the relaxation amplitude $\Delta r(\infty, 0)=r(\infty)-r(0)$ is the difference between the reluctivity in the equilibrium state at $t=\infty$ and immediately after demagnetization at $t=0$. Introducing a box-type distribution [12] in the logarithm of $\tau$ instead of one discrete relaxation time is necessary to describe the experimental data in most cases. According to eq. (2) this is equivalent to a box-type distribution in the activation enthalpy, $Q-\Delta Q \leq Q \leq Q+\Delta Q$, with a width $\Delta Q$. The relaxation function is then given by

$$
G(t, \tau)=1+\frac{\operatorname{Ei}\left(-t / \tau_{1}\right)-\operatorname{Ei}\left(-t / \tau_{2}\right)}{\ln \left(\tau_{2} / \tau_{1}\right)}
$$

where Ei denotes the exponential integral [16].

The relaxation strength of the MAE may be estimated by considering the micro-magnetic model of a ferromagnet divided into domains. The domains are separated by Bloch walls which lay in a potential, that consists of a static part $\phi_{\circ}$ corresponding to the interaction with all immobile lattice defects, e.g. dislocations, and a time dependent part $\phi_{\mathrm{r}}(t)$ due to mobile defects, e.g. carbon or nitrogen atoms. The increase of the magnetization $\Delta J$ by a displacement $U$ of the domain wall and the magnetostatic force $P$ due to the applied field $H$ are given by

$$
\Delta J=p J_{\mathrm{s}} U S \text { and } P=\mu_{\mathrm{o}} p J_{\mathrm{s}} H,
$$

where $p=2 \cos \varphi \cos \theta$ is a geometry factor, with $\varphi$ and $\theta$ the angle of the domain wall normal to the applied field and to the magnetization, respectively. $S$ corresponds to the domain wall surface per unit volume and $J_{\mathrm{s}}$ to the saturation magnetization. In this case of small displacements, i.e. small applied fields, the reluctivity can be derived, according to Kronmüller [9, 10], to

$$
r(t)=\frac{R_{\circ}+R_{\mathrm{r}}(t)}{\mu_{\mathrm{o}} p^{2} J_{\mathrm{s}}^{2} S},
$$

where $R_{\circ}$ and $R_{\mathrm{r}}(t)$ are related to the second derivatives of the wall potentials $\phi_{\mathrm{o}}$ and $\phi_{\mathrm{r}}(t)$, respectively. $R_{\mathrm{o}}$ and $R_{\mathrm{r}}(\infty)$ are, respectively, the unrelaxed initial reluctivity and a relaxation constant, depending on the type of the domain wall and defect symmetry, which can be expressed $[9,17]$ as

$$
R_{\mathrm{o}}=\frac{2 \pi^{2} \lambda \sigma_{\mathrm{o}} \delta_{\mathrm{o}}}{l_{\sigma}^{2}} \quad \text { and } \quad R_{\mathrm{r}}(\infty)=\frac{2 \pi^{2} N_{\mathrm{i}} \epsilon^{2}}{3 \delta_{\mathrm{o}} k T},
$$

where $\lambda$ is the magnetostriction constant, $\sigma_{\mathrm{o}}$ the internal stress amplitude, $\delta_{\mathrm{o}}$ the domain wall parameter, $l_{\sigma}$ the wave length of the spatial variation of $\sigma_{\mathrm{o}}$, and $N_{\mathrm{i}}$ the number of interstitial atoms. For a concentration $C_{\mathrm{i}}=1$ at.ppm of interstitials, $N_{\mathrm{i}} / N=10^{-6}$, where $N$ is the number of iron atoms per unit volume. Owing to the exponential time law, eq. (3), the relaxation strength between $t=0$ and $\infty$ is given by

$$
\frac{\Delta r(\infty, 0)}{r(0)}=\frac{R_{\mathrm{r}}(\infty)}{R_{\mathrm{o}}}=\left(\frac{l_{\sigma}}{\delta_{\mathrm{o}}}\right)^{2} \frac{N \epsilon^{2}}{3 k T \lambda \sigma_{\mathrm{o}}} C_{\mathrm{i}}
$$


To estimate the relaxation amplitude the following values of the intrinsic parameters are used: $N=8.5 \times 10^{28} \mathrm{~m}^{-3}, \lambda=20 \times 10^{-6}, \sigma_{\mathrm{o}}=5 \times 10^{8} \mathrm{Nm}^{-2}, \epsilon_{\mathrm{C}}=8.4 \times 10^{-23} \mathrm{~J}$ and $T=270 \mathrm{~K}$ or $\epsilon_{\mathrm{N}}=5.7 \times 10^{-23} \mathrm{~J}$ and $T=255 \mathrm{~K}$ for carbon or nitrogen [18], respectively. The values of $l_{\sigma}$ and $\delta_{0}$ depend strongly on the microstructure, e.g. dislocation density or grain size, and typical values characteristic for well-annealed high-purity iron were used, i.e. $l_{\sigma} / \delta_{0} \approx 50$. Specific factors for the relaxation strengths can be obtained to

$$
\frac{\Delta r(\infty, 0)}{r(0)} / C_{\mathrm{C}}=1.1 \times 10^{-2} \text { at.ppm }^{-1} \quad, \quad \frac{\Delta r(\infty, 0)}{r(0)} / C_{\mathrm{N}}=0.5 \times 10^{-2} \text { at.ppm }^{-1}
$$

\section{MEASURING TECHNIQUE}

The magnetic after-effect was measured via the time-dependent change of the reluctivity $r(t)$ after demagnetizing the specimen. The measurements are performed in a fully automated a.c. measuring device [19] where the specimen is centered in the coil of a voltage-controlled LC oscillator. The resonance frequency of this LC circuit can be given in good approximation by

$$
\nu(t)=\frac{1}{2 \pi} \sqrt{\frac{1}{L(t) C}-\left(\frac{R_{\mathrm{o}}}{L(t)}\right)^{2}},
$$

where $C$ is the capacity, $L(t)$ and $R_{\mathrm{o}}$ are the inductance and ohmic resistance of the coil, respectively. For a long coil, i.e. length $l$ much larger than cross-sectional area $A_{c}$, the inductance is given by

$$
L_{s}(t)=\mu_{\circ} \mu_{s}(t) n^{2} \frac{A_{c}}{l},
$$

where $\mu_{s}$ is the permeability of the sample and $n$ the number of turns of the coil. If the coil is only partially filled by the sample, the magnetic flux though the cross-sectional area is given by

$$
\phi(t)=\vec{B}(t) \cdot \vec{A}_{c}=\left[\mu_{s}(t) \vec{A}_{s}+\left(\vec{A}_{c}-\vec{A}_{s}\right)\right] \mu_{\mathrm{o}} \vec{H}=\vec{\mu}(t) \mu_{\mathrm{o}} \vec{A}_{c} \vec{H} .
$$

where $A_{s}$ is the cross-sectional area of the sample and $\bar{\mu}(t)$ the measured time-dependent permeability. The permeability of the specimen yields

$$
\mu_{s}(t)=(\bar{\mu}(t)-1) \frac{A_{c}}{A_{s}}+1 \quad \text { with } \quad \bar{\mu}(t)=\frac{L_{s}(t)}{L_{c}},
$$

where $L_{c}$ and $L_{s}(t)$ are the inductances of the empty coil and the coil including the specimen, respectively. In addition, an oscillating electromagnetic field entering a conducting medium is damped by the so-called skin effect to $1 / \mathrm{e}$ of its initial amplitude at a distance

$$
\delta=\left(\sigma \mu_{\text {stat }} \mu_{\mathrm{o}} \pi \nu\right)^{-1 / 2}
$$

which is called the skin depth and depends on the conductance $\sigma$ and static permeability $\mu_{\text {stat }}$ of the specimen, and on the frequency $\nu$ of the applied field. This skin effect further reduces the cross-sectional area of the sample. To a first approximation, this may be corrected by a factor $f_{\text {skin }}$ (for more detailed description see [9,20-22]). Using eqs. (10) and (13), the susceptibility of the specimen can be written as

$$
\chi_{s}(t)=\mu_{s}(t)-1=\left(\frac{\nu_{\mathrm{o}}^{2}}{\nu^{2}(t)} \cdot \frac{1+\sqrt{1-\left(4 \pi C R_{\mathrm{o}} \nu(t)\right)^{2}}}{1+\sqrt{1-\left(4 \pi C R_{\mathrm{o}} \nu_{\mathrm{o}}(t)\right)^{2}}}-1\right) \cdot \frac{A_{c}}{A_{s}} \cdot f_{\text {skin }}
$$

where $\nu$ and $\nu_{0}$ are the resonance frequencies with and without specimen, respectively. The MAE results are represented as relative relaxation amplitudes

$$
\frac{\Delta r\left(t_{1}, t_{2}, T\right)}{r\left(t_{1}, T\right)}=\frac{r\left(t_{2}, T\right)-r\left(t_{1}, T\right)}{r\left(t_{1}, T\right)},
$$

where in the present measurements $t_{1}=0.5 \mathrm{~s}$ and $t_{2}$ is varied from 1.5 to $179.5 \mathrm{~s}$. Using eq. (15), this representation has the advantage that both the filling factor $A_{c} / A_{s}$ and the skin depth correction $f_{\text {skin, }}$, which are difficult to determine, cancel out and can be ignored. 


\section{EXPERIMENTAL RESULTS}

\subsection{Specimen Preparation and Characterization}

Polycrystalline high-purity iron from various suppliers was additionally purified by annealing in Pd-diffused $\mathrm{H}_{2}$ atmosphere at $1000 \mathrm{~K}$ for $70 \mathrm{~h}$ and subsequently degassed in high vacuum at $950 \mathrm{~K}$ for $20 \mathrm{~min}$. Typically, these specimens had residual resistivity ratios of $R R R \approx 1600$ and comparable grain structures or textures. The specimens were doped to various carbon or nitrogen contents by heating in controlled mixtures of $\mathrm{CH}_{4}-\mathrm{H}_{2}$ or $\mathrm{NH}_{3}-\mathrm{H}_{2}$ atmospheres, respectively. The carbon and nitrogen contents were found to lie in the range between 40 and 250 at.ppm as determined by high-temperature combustion and Kjeldahl technique.

In addition, we analysed high-purity iron, which was produced by electrolytic deposition [23]. In order to avoid the agglomeration of carbon atoms and carbide formation, the specimens were heated in vacuum at $1073 \mathrm{~K}$ for $10 \mathrm{~min}$ and then quenched, which will be referred to as-produced. Some specimens were further purified by annealing at $1073 \mathrm{~K}$ first in wet and then in dry $\mathrm{H}_{2}$ atmospheres for $7 \mathrm{~h}+6 \mathrm{~h}$ or $24 \mathrm{~h}+24 \mathrm{~h}$, respectively, later referred to $\mathrm{H}_{2}$-purified. A chemical analysis by means of combustion-infrared absorbtion yielded carbon and nitrogen contents given in table 1, together with the residual resistivity ratios (RRR), which were measured at $4.2 \mathrm{~K}$ and $293 \mathrm{~K}$ under an applied magnetic field of $0.1 \mathrm{~T}$.

\subsection{Magnetic After-Effect Measurements}

Carbon and nitrogen doped high-purity iron show relaxation maxima at about $265 \mathrm{~K}$ and $250 \mathrm{~K}$, respectively, which can be described by the time law of the Richter MAE [19,22,24]. For C or N contents up to 250 at.ppm the relaxation amplitude of the corresponding maximum depends linearly on the interstitial concentration as determined by chemical analysis. Calibration factors of the Richter MAE for C and $\mathrm{N}$ can be obtained, which are in good agreement with the factors estimated by the theoretical considerations, see eq. (9), and with results of internal friction measurements on identically prepared specimens [22].

In the following, the general analytical features of the MAE technique will be explained by results on electrolytically-produced iron. For the as-produced specimen, the MAE spectrum in the temperature range of the $\mathrm{C}$ and $\mathrm{N}$ Richter MAE is shown in Fig. 1. The large relaxation maximum at $265 \mathrm{~K}$ with an amplitude of $76 \%$ indicates the presence of $(76 \pm 10)$ at.ppm carbon, see eq. (9). A numerical analysis according to the orientation MAE, eqs. (2)-(4), yields a mean activation enthalpy $Q=(0.86 \pm 0.02) \mathrm{eV}$ with a box-type distribution of width $\Delta Q=0.03 \mathrm{eV}$ and a pre-exponential factor $\tau_{\mathrm{o}}=10^{-15 \pm 0.5} \mathrm{~s}$. No indication for any nitrogen is found, i.e. neither any shoulder in the MAE around $250 \mathrm{~K}$, nor any activation enthalpy below $0.83 \mathrm{eV}$. Both $\mathrm{H}_{2}$-purified specimens $(7 \mathrm{~h}+6 \mathrm{~h}$ and $24 \mathrm{~h}+24 \mathrm{~h}$ ) show a nearly temperature-independent relaxation background but no maximum in this temperature region (Fig. 2) indicating a $\mathrm{C}$ and $\mathrm{N}$ content of below 0.1 at.ppm, which is approximately the sensitivity limit. The difference to the chemical analysis (Tab. 1) may be due to carbide precipitates, since only interstitially dissolved $\mathrm{C}$ gives rise to the MAE.

Additionally, the as-produced specimen was firstly measured up to $400 \mathrm{~K}$ and in a second run up to

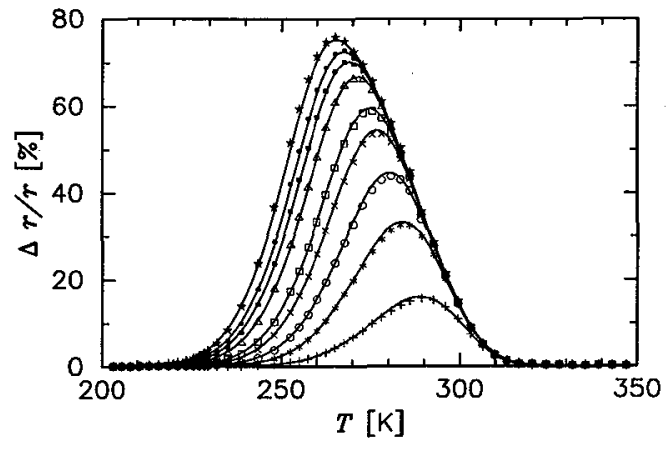

Figure 1: MAE spectrum of as-produced iron. The experimental data are represented by symbols and the solid lines correspond to a numerical fit to the time law of the Richter MAE, eqs. (2)-(4), indicating the presence of carbon.

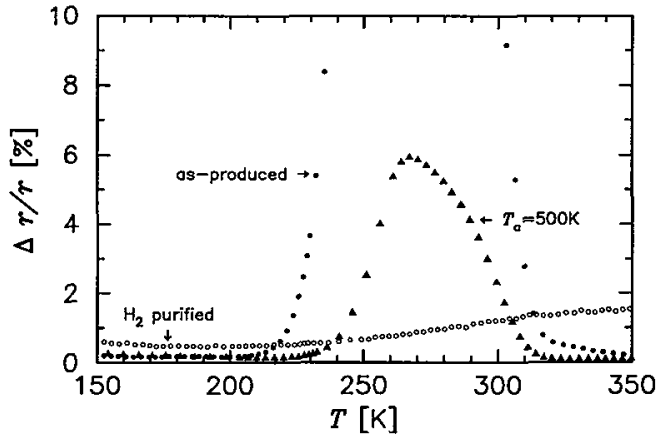

Figure 2: MAE spectrum of as-produced ( $\bullet$ ), as-produced $T_{\mathrm{a}}=500 \mathrm{~K}(\boldsymbol{\Lambda})$, and $\mathrm{H}_{2}$-purified (o) iron. The carbon Richter MAE maximum of the as-produced specimen reaches up to $76 \%$ (see Fig. 1). 


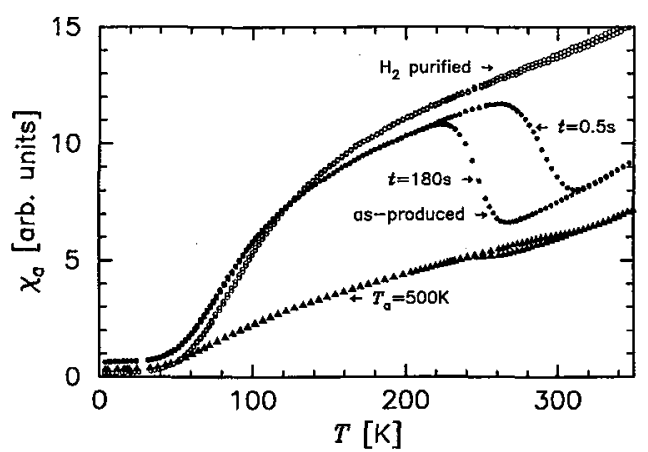

Figure 3: Initial susceptibility $\chi_{\mathrm{a}}$ as a function of temperature $T$ for as-produced ( $\bullet$ ), as-produced $T_{\mathrm{a}}=500 \mathrm{~K}(\boldsymbol{\Lambda})$, and $\mathrm{H}_{2}$ purified ( 0 ) iron. The two curves for each specimen represent values $0.5 \mathrm{~s}$ (upper) and $180 \mathrm{~s}$ (lower) after demagnetization, as indicated for the Richter MAE (as-produced).

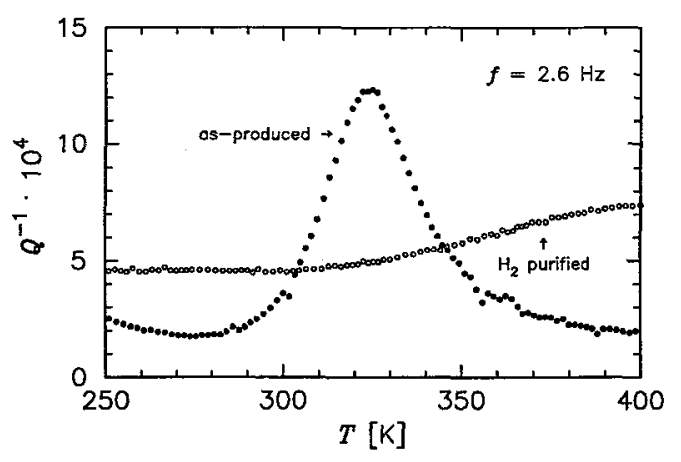

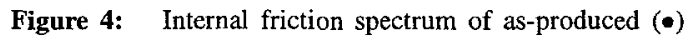
and $\mathrm{H}_{2}$-purified (o) iron. Resonance frequency in the Snoek maximum of carbon $f=2.6 \mathrm{~Hz}$.

$500 \mathrm{~K}$, equivalent to an annealing at $T_{\mathrm{a}}=400$ and $500 \mathrm{~K}$, respectively, for about $20 \mathrm{~min}$. After $T_{\mathrm{a}}=400 \mathrm{~K}$ the relaxation strength is decreased to $74 \%$ and after $T_{\mathrm{a}}=500 \mathrm{~K}$ drastically to $6 \%$ (Fig. 2), indicating that between 400 and $500 \mathrm{~K}$ carbon undergoes long-range diffusion, agglomerates and forms carbide precipitates. A similar precipitation behaviour was observed in nitrogen doped iron [24].

\subsection{Initial Susceptibility}

The $\mathrm{AC}$ susceptibility of $\mathrm{H}_{2}$-purified high-purity iron, determined according to eq. (15) but without filling factor and skin depth corrections, strongly decreases for low temperatures (Fig. 3), owing to the increase in conductance and subsequent decrease in skin depth. From the plateau value at $4.2 \mathrm{~K}$, the residual resistivity of the specimen can be estimated [20], which allows to obtain further information about the impurity level. Additionally, the as-produced specimen shows the typical time-dependant decrease between 220 and $310 \mathrm{~K}$ (Fig. 3), caused by the decrease of the Bloch wall mobility due to the Richter MAE of carbon.

\subsection{Internal Friction Measurements}

For comparison, the anelastic relaxation of electrolytically-produced high-purity iron was measured by internal friction applying an inverse torsion pendulum described in [6]. Fig. 4 shows the internal friction spectrum in the range of the Snoek maxima for $\mathrm{C}$ and $\mathrm{N}$. The as-produced specimen shows a large relaxation maximum at about $323 \mathrm{~K}$ with the height of $Q^{-1}=10.7 \times 10^{-4}$ (background subtracted) corresponding to a carbon content of $(75 \pm 10)$ at.ppm, where the uncertainty is due to the unknown structure factor. Both $\mathrm{H}_{2}$-purified specimens show only an increased background but no maximum, indicating a $\mathrm{C}$ and $\mathrm{N}$ content of below 0.5 at.ppm, which agrees well with the MAE results.

\begin{tabular}{|l|c|c|c|c|c|c|c|}
\hline Specimen & \multicolumn{2}{|c|}{$\begin{array}{c}\text { chem. analysis } \\
\text { C }\end{array}$} & $\begin{array}{c}\text { RRR } \\
\text { [at.ppm] }\end{array}$ & \multicolumn{2}{c|}{ MAE meas. } & \multicolumn{2}{c|}{ IF meas. } \\
& [at.ppm] & $\rho_{4.2 \mathrm{~K}} / \rho_{293 \mathrm{~K}}$ & $\Delta r / r$ & $\mathrm{C}(\mathrm{rel}$.) & $Q^{-1}$ & $\mathrm{C}$ (rel.) \\
{$[\%]$} & {$[$ at.ppm] } & {$\left[10^{-4}\right]$} & {$[$ at.ppm] } \\
\hline as-produced & $97.2 \pm 0.5$ & $1.2 \pm 0.4$ & 107 & 76 & $76 \pm 10$ & 10 & $75 \pm 10$ \\
$\mathrm{H}_{2}$-purified 7 $\mathrm{h}+6 \mathrm{~h}$ & $7.0 \pm 0.5$ & $0.4 \pm 0.4$ & 1040 & 0 & $<0.1$ & 0 & $<0.5$ \\
$\mathrm{H}_{2}$-purified $24 \mathrm{~h}+24 \mathrm{~h}$ & $6.5 \pm 0.5$ & $0.4 \pm 0.4$ & 1200 & 0 & $<0.1$ & 0 & $<0.5$ \\
\hline
\end{tabular}

Table 1: $\mathrm{C}$ and $\mathrm{N}$ contents obtained by chemical analysis, residual resistivity ratios RRR, and results of MAE and internal friction measurements with estimated content of mobile carbon atoms, $\mathrm{C}$ (rel.).

\section{CONCLUSIONS}

a) MAE measurements are a non-destructive analysis method for low concentrations of interstitially dissolved carbon or nitrogen atoms allowing an extremely high sensitivity $(>0.1$ at.ppm). The long relaxation times enable an analysis at temperatures below the long-range carbon diffusion. 
b) The absolute values possess an uncertainty of about $10 \%$ due to the influence of the microstructure, e.g. dislocation density or grain size. This error can be improved by the calibration of one typical specimen with a quantitative method, e.g. chemical analysis.

c) Additionally, the method offers a fast and very precise way to determine the activation enthalpy and pre-exponential factor of interstitial diffusion.

d) In-situ annealing studies are possible and allow to detect the formation of precipitates.

e) At low temperature the $\mathrm{AC}$ susceptibility offers an estimation of the residual resistivity and hence an additional possibility to check the impurity content.

\section{Acknowledgements}

The authors are very grateful to Prof. K. Abiko and Dr. S. Takaki, Tohoku University, Sendai, for supplying the chemically-analysed electrolytic high-purity iron and to Mr. R. Henes for the RRR measurements.

\section{References}

[1] Garten R.P.H. and Tölg G., "How accurate is the analytical characterization of high purity metals?", Ultra High Purity Base Metals, Kitakyushu-City 24-27 May 1994, K. Abiko, K. Hirokawa and S. Takaki Eds. (The Japan Institute of Metals, Sendai, 1995) pp. 191-209.

[2] Lawrenz D., "The determination of C, S, O, N and H in high purity base metals", in [1] pp. 183-190.

[3] Fédoroff M. and Rouchard J.-C., "Recent progress in the analysis of high-purity metals", in [1] pp. 210-216.

[4] Nowick A.S. and Berry B.S., Anelastic Relaxation in Crystalline Solids (Academic Press, New York, 1973).

[5] Weller M., J. Phys. 46 C10 (1985) 7-14.

[6] Weller M., "Characterization of high purity bcc metals by mechanical spectroscopy" this conference.

[7] Seeger A., Kronmüller H., and Rieger H., Z. angew. Phys. 18 (1965) 377-395.

[8] Seeger A., Magnetismus - Struktur und Eigenschaften magnetischer Festkörper (VEB Deutscher Verlag für Grundstoffindustrie, Leipzig, 1967) pp. 160-175.

[9] Kronmüller H., Nachwirkung in Ferromagnetika (Springer, Berlin, 1968).

[10] Kronmüller $\mathrm{H}$., "Studies of point defects in metals by means of mechanical and magnetic relaxation", Vacancies and Interstitials in Metals, Jülich 23-28 September 1968, A. Seeger, D. Schumacher, W. Schilling, and J. Diehl Eds. (North-Holland, Amsterdam, 1970) pp. 667-727.

[11] Kronmüller H., Hydrogen in Metals I, G. Alefeld and J. Völkl Eds. (Springer, Berlin - Heidelberg New York, 1978) pp. 289-320.

[12] Richter G., Ann. d. Physik 29 (1937) 605-635 and Ann. d. Physik 32 (1938) 683-700.

[13] Snoek J.L., Physica 5 (1938) 663-688, 6 (1939) 161-170, 591-592, 797-805, and 8 (1941) 711-733.

[14] De Vries G., Van Geest D.W., Gersdorf R., and Rathenaue G.W., Physica 25 (1959) 1131-1138.

[15] Néel L., J. Phys. Rad. 12 (1951) 339-351 and 13 (1952) 249-264.

[16] Abramowitz M. and Stegun I., Handbook of Mathematical Functions (National Bureau of Standards, Washington, 1972).

[17] Chikazumi S., Physics of Magnetism (John Wiley Sons Inc., New York, 1964).

[18] Bosman A.J., Investigation on magnetic after-effects due to interstitials (Ph.D. Thesis, University Amsterdam, 1960).

[19] Walz F., Appl. Phys. 3 (1974) 313-319 and phys. stat. sol. (a) 82 (1984) 179-189.

[20] Hirscher M., Schwendemann B., and Kronmüller H., Appl. Phys. A 41 (1986) 191-195.

[21] Walz F., phys. stat. sol. (a) 134 (1992) 509-520.

[22] Walz F., Weller M., and Hirscher M., to be published.

[23] Daicho T., Arisawa N., and Abiko K., "Refining of high purity electrolytic iron and its properties", in [1] pp. 397-402.

[24] Földeáki M., Kronmüller H., Schwendemann B., and Walz F., Z. f. Metallk. 73 (1982) 786-790. 